The quantity $K$ can also be written as

$$
\begin{aligned}
K & =\exp \left[-B\left(t_{0}\right) / G\left(t_{0}\right)\right] \\
& \times \int_{0}^{\infty} \frac{\exp \left\{\left[B\left(t_{0}\right) / G\left(t_{0}\right)\right] /\left[1+G\left(t_{0}\right) x\right]\right\} \exp (-\alpha x) d x}{1+G\left(t_{0}\right) x} .
\end{aligned}
$$

Next we utilize the well-known Taylor series expansion for the first exponential under the integral. After a straight forward change of variable one obtains

$$
\begin{aligned}
K=\frac{\exp \left[-B\left(t_{0}\right) / G\left(t_{0}\right)\right]}{G\left(t_{0}\right)} \sum_{l=0}^{\infty}\left(\frac{\left[B\left(t_{0}\right) / G\left(t_{0}\right)\right]^{l}}{l !}\right. \\
\left.\quad \times \int_{0}^{\infty} \frac{\exp \left\{-\left[\alpha / G\left(t_{0}\right)\right] U\right\}}{(1+U)^{l+1}} d U\right) .
\end{aligned}
$$

The integral which appears in (A5) has been evaluated in Ref. 28, and this leads to

$$
\begin{gathered}
K=\frac{\exp \left[-B\left(t_{0}\right) / G\left(t_{0}\right)\right]}{G\left(t_{0}\right)} \sum_{l=0}^{\infty}\left[\frac{\left[B\left(t_{0}\right) / G\left(t_{0}\right)\right]^{l}}{l !}\right. \\
\times(l !)^{-1} \sum_{k=1}^{l}(k-1) !\left(\frac{-\alpha}{G\left(t_{0}\right)}\right)^{l-k}-\frac{\left[\alpha / G\left(t_{0}\right)\right]^{l}}{l !} \\
\left.\times \exp \left(\frac{\alpha}{G\left(t_{0}\right)}\right) E i\left(\frac{-\alpha}{G\left(t_{0}\right)}\right)\right],
\end{gathered}
$$

where $E i(z)=$ exponential integral function.

Equation (3.7) is now obtained from (A6) and the following series expansion for the Bessel function ${ }^{29}$ :

$$
J_{0}\left(\frac{2\left[B\left(t_{0}\right) \alpha\right]^{1 / 2}}{G\left(t_{0}\right)}\right)=\sum_{l=0}^{\infty} \frac{(-1)^{l}\left\{2\left[B\left(t_{0}\right) \alpha\right]^{1 / 2} / G\left(t_{0}\right)\right\}^{2 l}}{2^{2 l}(l !)^{2}} .
$$

${ }^{28}$ I. S. Gradshteyn and I. M. Ryzhik, Ref. 18, (Eq. 3.353-2) on p. 311.

${ }^{29}$ I. S. Gradshteyn and I. M. Ryzhik, Ref. 18 (Eq. 8.441-1), p. 959 .

\title{
Focusing of a Relativistic Electron Flow*
}

\author{
H. C. Hsien AND J. E. Rowe \\ Department of Electrical Engineering, The University of Michigan, Ann Arbor, Michigan
}

(Received 7 February 1968)

\begin{abstract}
The trajectory differential equation governing the motion of relativistic electrons is derived in terms of the scalar and vector potentials of the system using the principle of least action. The conservation of energy and momentum are used to develop the paraxial-ray differential equation describing the beam radius of a laminar-flow relativistic electron beam. The focusing of the electron beam in drift and accelerating regions has been examined and the conditions for perfect balancing and nonspreading of a laminar-flow drifting beam are also derived. It is shown that the equilibrium condition for Brillouin flow is that $2 \omega_{L}{ }^{2}-\omega_{p}^{2}\left[1-\left(u_{z} / c\right)^{2}\right]=0$, where $\omega_{L}$ is the Larmor precession frequency and $\omega_{p}$ the electron-plasma frequency. $u_{z}$ and $c$ denote, respectively, the axial beam velocity and the speed of light in vacuum. The variation of the normalized ripple amplitude and the scallop wavelength of a drifting beam is discussed. The profile of a beam accelerated in a uniform longitudinal electrostatic field is also illustrated.
\end{abstract}

\section{INTRODUCTION}

The subject of electron-beam focusing has been investigated extensively by various workers ${ }^{1-11}$ in-

* This work was supported by the National Aeronautics and Space Administration.

${ }^{1} \mathrm{~J}$. R. Pierce, Theory and Design of Electron Beams (D. Van Nostrand, Inc., New York, 1949).

2 P. E. Strel'nikov et al., Sov. Phys.-Tech.Phys. 6, 285 (1961).

${ }^{3}$ K. K. N. Chang, Proc. IRE 43, 62 (1955).

4 P. K. Tien, J. Appl. Phys. 25, 1281 (1954).

${ }^{5}$ A. M. Clogston and H. Heffner, J. Appl. Phys. 25, 436 (1954).

6 E. R Harrison, J. Electron. Control 4, 193 (1958).

7 I. N. Meshkov and B. V. Chirikov, Sov. Phys.-Tech. Phys. 10,1688 (1966)

8 J. D. Lawson, J. Electron. Control 5, 146 (1958).

9 W. H. Bennett, Phys. Rev. 98, 1584 (1955).

${ }^{10} \mathrm{~W}$. H. Bennett, Phys. Rev. 45, 890 (1935).

${ }^{11}$ S. E. Graybill and S. V. Nablo, Appl. Phys. Letters 8, 18 (1966). terested in their use, e.g., in microwave tubes and linear electron accelerators. In the dynamic analysis of electron beams encountered in most microwave beam-type devices $^{1-6}$ relativistic effects are usually negligible, whereas, in the focusing of charged particles in a linear electron accelerator, relativistic effects (selffocusing and pinching) play a primary role. Recently the focusing of a high-intensity electron beam in an accelerating tube has been discussed theoretically by Meshkov and Chirikov, ${ }^{7}$ and previously the magnetic self-focusing of partially neutralized relativistic electron beams drifting in the absence of any longitudinal field has been analyzed theoretically for a variety of idealized stream conditions $s^{8-10}$ and observed experimentally. ${ }^{11}$

It appears that little attention has been given to the 
study of the dynamics of a relativistic electron flow under the influence of its own fields in the presence of externally applied static electric and magnetic fields. The purpose of the present study is to investigate the focusing of a relativistic cylindrical electron beam in the presence of applied static axial electric and magnetic fields, assuming that the effects of radiation and collisions are negligible, and further that the transverse velocity is small in comparison with the axial velocity. The principle of "least action" is used in deriving the trajectory differential equation of the charged particle. ${ }^{12}$

\section{TRAJECTORY DIFFERENTIAL EQUATIONS}

The canonical momentum $\mathbf{p}$ of an electron moving at a relativistic velocity $\mathbf{u}$ may be written as

$$
\mathbf{p}=m \mathbf{u}+e \mathbf{A},
$$

where $m=m_{0} /\left[1-(u / c)^{2}\right]^{1 / 2}$ and $m_{0}$ and $e$ are, respectively, the rest mass and charge (negative quantity) of an electron, $c$ denotes the speed of light in vacuum, A is the magnetic vector potential, and $u$ denotes the magnitude of the velocity vector $\mathfrak{u}$.

The principle of least action is conveniently written as

$$
\delta \int_{x_{\alpha 1}}^{x_{\alpha \mathbf{q}}} \mathbf{p} \cdot d \mathbf{l}=\int_{x_{\alpha_{1}}}^{x_{\alpha 2}}(m \mathfrak{u}+e \mathbf{A}) \cdot d \mathbf{l}=0,
$$

where 1 is the position vector, $x_{\alpha_{1}}$ and $x_{\alpha_{2}}$ denote the coordinates of the end points of the line integral, and $\delta$ is the usual variational operator. By taking one of the three spatial coordinates, e.g., $z$, as the independent variable, and defining $r^{\prime} \equiv d r / d z$ and $\theta^{\prime} \equiv d \theta / d z$ in a cylindrical coordinate system, Eq. (2) can be written as follows:

$$
\begin{aligned}
\delta \int_{z 1}^{z_{2}}\left\{m u\left[r^{\prime 2}+\left(r \theta^{\prime}\right)^{2}+1\right]^{1 / 2}\right. \\
\left.+e\left(A_{r} r^{\prime}+A_{\theta} r \theta^{\prime}+A_{z}\right)\right\} d z=0 .
\end{aligned}
$$

The Eulerian equations for this system will yield the differential equations for the electron orbit provided that the magnitude of the velocity $u$ is known as a function of $z$. Since, according to the principle of least action, the varied path satisfies the law of conservation of energy, $u$ can be expressed in terms of the kinetic energy $T$ and the rest energy $\epsilon_{0}$ of the particle, i.e.,

$$
(u / c) /\left[1-(u / c)^{2}\right]^{1 / 2}=\left(T^{2}+2 T \epsilon_{0}\right)^{1 / 2} / \epsilon_{0},
$$

since $m c^{2}=\epsilon_{0}+T$. Thus, after introducing Eqs. (1) and (4), the variational equation, Eq. (3), can be written as follows:

$$
\delta \int_{z_{1}}^{z_{1}} P d z=0
$$

12 W. Panofsky and M. Phillips, Classical Electricity and Magnetism (Addison-Wesley Publishing Co., Inc., Cambridge, Mass, 1955), Chap. 23. where $P \equiv\left(\tau^{2}+2 \tau\right)^{1 / 2}\left(r^{\prime 2}+r^{2} \theta^{\prime 2}+1\right)^{1 / 2}+\left(e c / \epsilon_{0}\right)\left(A_{r} r^{\prime}+\right.$ $\left.A_{\theta} r \theta^{\prime}+A_{z}\right)$ with $\tau \equiv\left(T / \epsilon_{0}\right)$ and $\epsilon_{0} \equiv m_{0} c^{2}$. Then the Eulerian equations for $r(z)$ and $\theta(z)$ can be obtained from

and

$$
(d / d z)\left(\partial P / \partial r^{\prime}\right)-(\partial P / \partial r)=\mathbf{0}
$$

$$
(d / d z)\left(\partial P / \partial \theta^{\prime}\right)-(\partial P / \partial \theta)=0 .
$$

For an axially symmetric system, $\partial P / \partial \theta=0$ and Eqs. (5) and (6) are combined to give

$$
\left\{\left(\tau^{2}+2 \tau\right)^{1 / 2}\left(r^{2} \theta^{\prime}\right) /\left[r^{\prime 2}+\left(r \theta^{\prime}\right)^{2}+1\right]^{1 / 2}\right\}+\left(e c / \epsilon_{0}\right) A_{\theta} r=M_{0},
$$

where $M_{0}$ is a constant of integration, independent of $z$. It is observed that Eq. (7) expresses the conservation of the $\theta$ component of the canonical momentum since

$$
\left(r \theta^{\prime}\right) /\left(r^{\prime 2}+r^{2} \theta^{\prime 2}+1\right)^{1 / 2}=(r \dot{\theta}) / u,
$$

where the dot denotes the time derivative. It is easily shown that Eq. (7) is equivalent to the following familiar relationship:

$$
r p_{\theta}+e A_{\theta} r=K_{0},
$$

in which $p_{\theta}=m_{0}(r \dot{\theta}) /\left[1-(u / c)^{2}\right]^{1 / 2}$ and $K_{0} \equiv m_{0} c M_{0}$. A differential equation for $r(z)$ can be obtained by combining Eqs. (5), (6a), and (7):

$$
\begin{aligned}
r^{\prime \prime}- & (R / 2 w)\left[(\partial w / \partial r)-r^{\prime}(\partial w / \partial z)\right] \\
= & \left(-R^{3 / 2} / w^{1 / 2}\right)\left(e c / \epsilon_{0}\right)\left[\left(\partial A_{r} / \partial z\right)-\left(\partial A_{z} / \partial r\right)\right],
\end{aligned}
$$

where $R \equiv\left(1+r^{\prime 2}\right), w \equiv \xi\left(1-\eta^{2}\right), \xi \equiv\left(\tau^{2}+2 \tau\right)$, and

$$
\eta \equiv \xi^{-1 / 2}\left[\left(M_{0} / r\right)-\left(e c A_{\theta} / \epsilon_{0}\right)\right] .
$$

In view of the fact that $w$ is a function of $\xi$ and $\eta$, and $\xi$ depends in turn on $\tau$, which is the ratio of the particle kinetic energy $T$ to the rest energy $\epsilon_{0}$, once $T(r, z)$ and $\mathbf{A}(r, z)$ are known, then Eq. (10) can be solved for $r(z)$ for a properly specified set of input conditions; $\theta(z)$ then can be determined directly from Eq. (7).

It should be noted that $\eta$ can be written as $\eta=$ $\left(u_{\theta} / u\right) \equiv r \dot{\theta} / u$, and $r^{\prime}$ as $r^{\prime}=u_{r} / u_{z} \equiv \dot{r} / \dot{z}$, where $u^{2}=$ $\left(u_{r}^{2}+u_{\theta}^{2}+u_{z}^{2}\right)$. If the radial velocity $u_{r}$ is much smaller than the axial velocity $u_{z}$, i.e., $r^{\prime 2} \ll 1$, then $R \approx 1$.

\section{PARAXIAL RAY EQUATIONS FOR AN AXIALLY SYMMETRIC FLOW}

Under the condition $r^{\prime 2} \ll 1$, Eq. (10) takes the following form:

$$
\begin{gathered}
r^{\prime \prime}=w^{-1}\left\{(\tau+1)\left[(\partial \tau / \partial r)-r^{\prime}(\partial \tau / \partial z)\right]+\eta(\xi)^{1 / 2}\left(M_{0} / r^{2}\right)\right. \\
\left.+\eta(\xi)^{1 / 2}\left(e c / \epsilon_{0}\right) \cdot\left[\left(\partial A_{\theta} / \partial r\right)-r^{\prime}\left(\partial A_{\theta} / \partial z\right)\right]\right\} \\
-w^{-1 / 2}\left(e c / \epsilon_{0}\right)\left[\left(\partial A_{r} / \partial z\right)-\left(\partial A_{z} / \partial r\right)\right] .
\end{gathered}
$$

The law of conservation of the particle energy can be expressed as

$$
T+e \Psi=\text { constant }
$$


so that

and

$$
\partial \tau / \partial r=\left(-e / \epsilon_{0}\right)(\partial \Psi / \partial r)
$$

$$
\partial \tau / \partial z=\left(-e / \epsilon_{0}\right)(\partial \Psi / \partial z) .
$$

In addition, if $\gamma$ and $\beta$ are defined as

$$
\gamma \equiv\left(1-\beta^{2}\right)^{-1 / 2} \text { and } \beta \equiv(u / c) \text {, }
$$

then from Eq. (4), $\xi=\left(\gamma^{2} \beta^{2}\right)$ so that $(\tau+1)=$ $(1+\xi)^{1 / 2}=\gamma$ and from Eqs. (9) and (11)

and

$$
\eta(\xi)^{1 / 2}=\left[\left(M_{0} / r\right)-\left(e c A_{\theta} / \epsilon_{0}\right)\right]=\gamma\left(u_{\theta} / c\right)
$$

$$
w=\xi\left(u_{z} / u\right)^{2} .
$$

As a result Eq. (12) can be written as

$$
\begin{aligned}
r^{\prime \prime}= & \left(1 / \gamma u_{z}^{2}\right)\left\{\left(-e / m_{0}\right)\left[(\partial \Psi / \partial r)-r^{\prime}(\partial \Psi / \partial z)\right]\right. \\
& -u_{\theta}(\partial / \partial r)\left[\left(M_{0} c / r\right)-\left(e / m_{0}\right) A_{\theta}\right]-\left(e / m_{0}\right) u_{\theta} r^{\prime} \\
& \left.\times\left(\partial A_{\theta} / \partial z\right)\right\}-\left(\gamma u_{z}\right)^{-1}\left(e / m_{0}\right)\left[\left(\partial A_{r} / \partial z\right)-\left(\partial A_{z} / \partial r\right)\right],
\end{aligned}
$$

where $\Psi(r, z)$ and $\mathbf{A}(r, z)$ are, respectively, the scalar and vector potentials of the system under consideration. The potentials, in general, consist of two parts (one part due to an external source and the other due to the electron stream space charge and current). Furthermore, these potentials must satisfy the following partial differential equations:

$$
\nabla^{2} \Psi=-\rho / \epsilon \text { and } \boldsymbol{\nabla} \times(\boldsymbol{\nabla} \times \mathbf{A})=\mu_{0} \rho \mathbf{u},
$$

where $\rho$ is the space-charge density and $\mu_{0}$ and $\epsilon$ denote the permeability and permittivity of vacuum, respectively.

For an axially symmetric system Poisson's equation becomes

$$
r^{-1}(\partial / \partial r)[r(\partial \Psi / \partial r)]+\left(\partial^{2} \Psi / \partial z^{2}\right)=-\rho_{0} \gamma / \epsilon
$$

and for a steady-state static condition $\boldsymbol{\nabla} \cdot \mathbf{A}$ can be taken as zero so that Eqs. (16) give, in component form,

$$
\begin{aligned}
& (\partial / \partial r)\left[r^{-1}(\partial / \partial r)\left(r A_{r}\right)\right]+\left(\partial^{2} A_{r} / \partial z^{2}\right)=-\mu_{0} \rho_{0} \gamma u_{r}, \\
& (\partial / \partial r)\left[r^{-1}(\partial / \partial r)\left(r A_{\theta}\right)\right]+\left(\partial^{2} A_{\theta} / \partial z^{2}\right)=-\mu_{0} \rho_{0} \gamma u_{\theta}
\end{aligned}
$$

and

$$
r^{-1}(\partial / \partial r)\left[r\left(\partial A_{z} / \partial z\right)\right]+\left(\partial^{2} A_{z} / \partial z^{2}\right)=-\mu_{0} \rho_{0} \gamma u_{z},
$$

where $\rho_{0}$ is the rest charge density which is a negative quantity for electrons and is assumed to be constant in the present investigation. It should be noted that, relativistically, charge density and current density are simply different aspects of the same thing. If $\rho_{0}$ is a "proper" charge density in a frame where charges are at rest, then $\rho=\gamma \rho_{0}$ gives the transformation from a charge density at rest to a charge density in a nonproper frame, ensuring the invariance of the total charge. This can be seen as follows: A spatial volume element $d v$ is related to a proper spatial volume $d v_{0}$ by $d v=d v_{0} / \gamma$, since only one dimension (e.g., the $z$ direction if $u_{z}$ is comparable to $c$ ) suffers a Lorentz contraction and hence $\rho d v=\rho_{0} d v_{0}$ and the charge within a given boundary remains invariant. The law of conservation of energy [Eq. (13)] can be written as

$$
m_{0} \gamma c^{2}+e \Psi=m_{0} c^{2}
$$

and the concept of the conservation of canonical momentum is expressed as

and

$$
\begin{gathered}
m_{0} \gamma u_{r}+e A_{r}=0, \\
m_{0} \gamma u_{\theta}+e A_{\theta}=K_{0} / r \\
m_{0} \gamma u_{z}+e A_{2}=0 .
\end{gathered}
$$

Combining Eqs. (17) and (18) yields

$$
\begin{array}{r}
r^{-1}(\partial / \partial r)[r(\partial \gamma / \partial r)]+\left(\partial^{2} \gamma / \partial z^{2}\right)=\beta_{0}{ }^{2} \gamma, \\
(\partial / \partial r)\left[r^{-1}(\partial / \partial r)(r U)\right]+\left(\partial^{2} U / \partial z^{2}\right)=\beta_{0}{ }^{2} U, \\
(\partial / \partial r)\left[r^{-1}(\partial / \partial r)(r V)\right]+\left(\partial^{2} V / \partial z^{2}\right)=\beta_{0}{ }^{2} V
\end{array}
$$

and

$$
r^{-1}(\partial / \partial r)[r(\partial W / \partial r)]+\left(\partial^{2} W / \partial z^{2}\right)=\beta_{0}{ }^{2} W,
$$

where

$$
\begin{gathered}
U \equiv\left(\gamma u_{r}\right), \quad V \equiv\left(\gamma u_{\theta}\right), \quad W \equiv\left(\gamma u_{z}\right), \\
\beta_{0}^{2} \equiv\left(\omega_{p}^{2} / c^{2}\right) \quad \text { and } \quad \omega_{p}^{2} \equiv\left(e \rho_{0} / m_{0} \epsilon\right) .
\end{gathered}
$$

Equations (19) are linear partial differential equations and can be solved by the standard technique of separation of variables. For example, it is easily observed that the general solution of Eqs. (19) can be written in product form $[R(r) Z(z)]$, where $R(r)$ denotes a linear combination of the $n$ th-order modified Bessel functions of the first kind, $I_{n}(\alpha r)$, the second kind, $K_{n}(\alpha r)$, where $\alpha \equiv\left(k^{2}+\beta_{0}^{2}\right)^{1 / 2}$, with $k$ being the separation constant. For $k \neq 0, Z(z)$ is a periodic sinusoidal function of $z$, whereas for $k=0$ it is a linear function of $z$. The solutions of Eqs. (19a) and (19d) involve the zero-order modified Bessel function, while that of Eqs. (19b) and (19c) involve the first-order modified Bessel function.

When a solid electron beam is considered, the quantities $\gamma, U, V$, and $W$ all must remain finite along the axis $r=0$, and consequently the modified Bessel function of the second kind is not permissible in the solution of Eqs. (19). Thus,

$$
\begin{gathered}
\gamma(r, \zeta)=M_{1} I_{0}(\alpha r) \cos k \zeta, \\
U(r, \zeta)=M_{3}(k / \alpha) I_{1}(\alpha r) \sin k \zeta, \\
V(r, \zeta)=M_{2} I_{1}(\alpha r) \cos k \zeta
\end{gathered}
$$

and

$$
W(r, \zeta)=M_{3} I_{0}(\alpha r) \cos k \zeta,
$$

in which $\boldsymbol{\nabla} \cdot \mathbf{A}=0$ has been used, and $\zeta \equiv\left(z-z_{0}\right) . M_{\mathbf{1}}$, 
$M_{2}, M_{3}$, and $z_{0}$ are the constants of integration which are yet to be determined.

From Eqs. (18) and (20), the potential in the beam region, allowing for the presence of applied static fields, can be expressed as follows:

$$
\begin{gathered}
\Psi(r, \zeta)=-E_{0} \zeta+\left(m_{0} c^{2} /-e\right) M_{1} I_{0}(\alpha r) \cos k \zeta+\left(m_{0} c^{2} / e\right) \\
A_{r}(r, \zeta)=\left(m_{0} /-e\right)(k / \alpha) M_{3} I_{1}(\alpha r) \sin k \zeta \\
A_{\theta}(r, \zeta)=\left(m_{0} /-e\right) M_{2} I_{1}(\alpha r) \cos k \zeta+\left(K_{0} / e r\right)
\end{gathered}
$$

and

$$
A_{z}(r, \zeta)=\left(m_{0} /-e\right) M_{3} I_{0}(\alpha r) \cos k \zeta,
$$

in which $\zeta=0$, i.e., $z=z_{0}$ denotes the entry into the interaction region under consideration.

It should be noted that any longitudinal inhomogeneity, which may exist in the system, is represented by the constant $k$. For example, in an infinitely long homogeneous beam $k$ can be taken as zero. For a constant velocity drifting beam, $E_{0}$ vanishes. In this case, $u_{z}=\left(M_{3} / M_{1}\right)$, which is independent of $z$. For an accelerating beam, $E_{0} \neq 0$ and it can easily be observed from Eqs. (18), (20), and (21) that $u_{z}$ does depend upon $z$. For a laminar flow, i.e., nonintersecting electron trajectories that are sufficiently well confined in the transverse direction, only the motion of the boundary electron need be considered. Thus, Eq. (15) can be used for the investigation of the variation of the radius of the beam boundary as a function of axial distance.

Let $y$ be the beam radius under consideration, and suppose that the quantity $(\alpha y)$ satisfies the following inequality:

$$
(\alpha y)^{4} \ll 1 .
$$

Then the Bessel functions may be expanded as follows:

and

$$
I_{0}(\alpha y) \approx 1+\frac{1}{4}(\alpha y)^{2}
$$

$$
I_{1}(\alpha y) \approx \frac{1}{2}(\alpha y)+\frac{1}{16}(\alpha y)^{3} .
$$

Thus upon substituting Eqs. (20) and (21) into Eq. (15), with the approximation (22), the following differential equation is obtained:

$$
\begin{gathered}
y^{\prime \prime}+y^{\prime}\left(\left(G_{2} / \cos k \zeta\right)\left[1-\frac{1}{4}\left(\alpha^{2} y^{2}\right)\right]+\left\{G_{1}{ }^{2} y^{2}\left[1-\frac{1}{4}\left(\alpha^{2} y^{2}\right)\right]\right.\right. \\
\left.\left.-\delta_{0}{ }^{2}\right\} k \tan k \zeta\right)+y\left[G_{1}{ }^{2}+\frac{1}{2}\left(\beta_{0}{ }^{2}-\alpha^{2} \delta_{0}{ }^{2}\right)\right] \\
+\frac{1}{16}\left(\alpha^{2} y^{3}\right)\left(\alpha^{2} \delta_{0}{ }^{2}-\beta_{0}{ }^{2}\right)=0
\end{gathered}
$$

where

$$
\begin{array}{r}
G_{2} \equiv\left[\left(e E_{0} / m_{0}\right)\left(M_{1} / M_{3}^{2}\right)\right], \quad G_{1} \equiv\left(\alpha M_{2} / 2 M_{3}\right), \\
\delta_{0} \equiv\left(c M_{1} / M_{3}\right) .
\end{array}
$$

$\omega_{p}$ is the electron-beam plasma angular frequency for a beam with infinite lateral extent. When the beam cross section is finite, such as is frequently the case, the plasma angular frequency is much smaller than that for an infinite beam due to the effect of the conducting boundary surrounding the beam. The effect of the finiteness of the beam size on the plasma frequency can be taken into account by replacing $\omega_{p}$ by $\omega_{q} \equiv$ $R_{p} \omega_{p}$, with $R_{p}$ denoting the plasma reduction factor. Formulas are available 'for the determination of $R_{p}$ in the case of a solid cylindrical beam passing through a metal tube. ${ }^{13}$ The constants $M_{1}, M_{2}$, and $M_{3}$ can be determined in terms of the physical and geometrical parameters specified at the input plane $\zeta=0$. It is obvious from Eqs. (20) and (21) that $M_{1}=\gamma_{0,0}=$ $\left[1+\left(-e \Psi_{0,0} / m_{0} c^{2}\right)\right]$, where $\Psi_{0,0}$ denotes the axial beam voltage at the input plane $\zeta=0$. From Eq. (20d), $M_{3}=\gamma_{0,0} u_{z}(0,0)=M_{1} u_{z, 0}$. If the axial beam current at the entrance plane is denoted by $\bar{I}_{0}$, then

$$
\begin{aligned}
\bar{I}_{0} & =\rho_{0} \int_{0}^{y_{0}} W 2 \pi r d r \\
& =\left(\pi y_{0}^{2}\right) \rho_{0} M_{3}\left[2 I_{1}\left(\alpha y_{0}\right) / \alpha y_{0}\right],
\end{aligned}
$$

where $y_{0}$ denotes the injection beam radius. In view of the fact that $\gamma_{0,0}=1 /\left[1-\left(u_{z, 0} / c\right)^{2}\right]^{1 / 2}$, specification of $\Psi_{0,0}$ determines $u_{z, 0}$ and $\gamma_{0,0}$ as well as $M_{1}$ and $M_{3}$. $\bar{I}_{0}$ and $y_{0}$ must also be specified in order to determine $\omega_{p}$. On the other hand, $M_{2}$ can be expressed in terms of the input $\theta$-component velocity, from Eq. (20c) as follows:

$$
M_{2} I_{1}\left(\alpha y_{0}\right)=\gamma\left(y_{0}, 0\right) u_{\theta}\left(y_{0}, 0\right)=M_{1} I_{0}\left(\alpha y_{0}\right) u_{\theta}\left(y_{0}, 0\right) .
$$

It is convenient to introduce a pitch angle parameter for the boundary electron at the input plane which is defined as

$$
\tan \psi_{0} \equiv u_{\theta}\left(y_{0}, 0\right) / u_{z}\left(y_{0}, 0\right)
$$

so that $G_{1}$ can be expressed as

$$
G_{1}=y_{0}^{-1} \tan \psi_{0} \text {. }
$$

Thus specification of the velocity ratio $\left(u_{\theta} / u_{z}\right)$ at $r=y_{0}$ and $\zeta=0$ determines the parameter $G_{1} ; \psi_{0}$ is small since $u_{\theta}$ is assumed to be much smaller than $u_{z}$.

It should be noted that the solution of Eq. (23) provides information on the profile of the electron beam in the accelerating region. Suppose that the normalized perturbation in beam radius, $Y$, and the normalized axial distance $x$ are respectively defined as

$$
Y \equiv\left(y-y_{0}\right) / y_{0} \text { and } x \equiv\left(\beta_{0} \zeta\right),
$$

where $y_{0}$ denotes the injection beam radius. Then Eq. (23) becomes

$$
\begin{gathered}
\left(d^{2} Y / d x^{2}\right)+(d Y / d x)\left\{\left(g_{2} / \cos \nu x\right)\left[1-\frac{1}{4} \sigma^{2}(1+Y)^{2}\right]\right. \\
\left.+\nu \tan \nu x\left[p_{0}^{2}(1+Y)^{2}-\frac{1}{4} \sigma^{2} p_{0}^{2}(1+Y)^{4}-\delta_{0}^{2}\right]\right\} \\
+(1+Y)\left[\left(p_{0}^{2} / \mu^{2}\right)+g_{3}\right]-(1+Y)^{3} \frac{1}{8} \sigma^{2} g_{3}=0,
\end{gathered}
$$

where

$$
\begin{array}{r}
g_{2}=a_{0}\left(\delta_{0} / \mu\right)\left(\delta_{0}^{2}-1\right)^{1 / 2}, \quad \sigma^{2}=\mu^{2}\left(1+\nu^{2}\right), \\
g_{3}=\frac{1}{2}\left[1-\left(1+\nu^{2}\right) \delta_{0}^{2}\right]
\end{array}
$$

${ }^{13}$ G. M. Branch and T. G. Mihran, IRE Trans. Electron Devices ED-2, 3 (1955). 


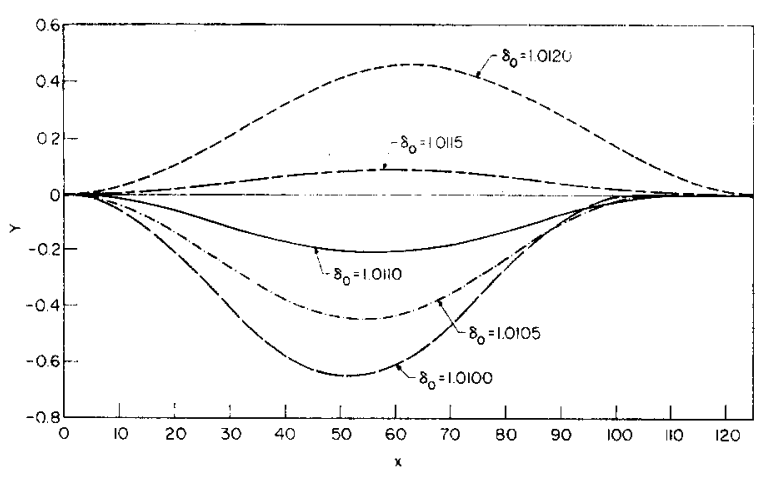

(a)

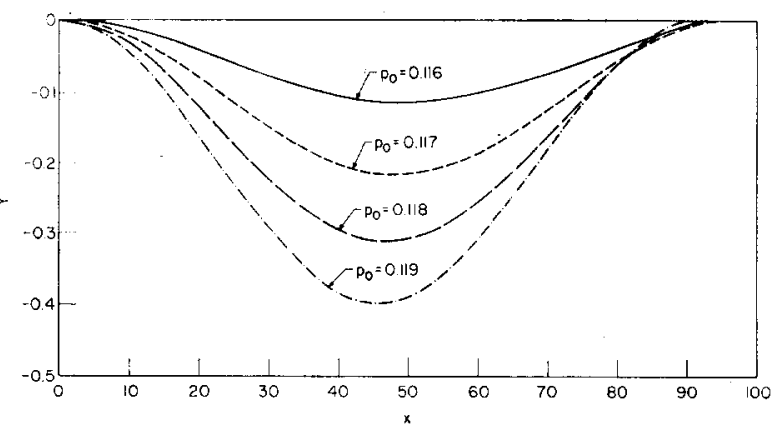

(b)

FIG. 1.(a) Velocity dependence of electron-beam profile when $Y^{2} \ll 1 .\left(\mu=1.0, p_{0}=0.1, \nu=a_{0}=0\right)$. (b) Effect of transverse velocities on electron-beam profile when $Y^{2} \ll 1$. $\left(\delta_{0}=1.015, \mu=1.0, \nu=a_{0}=0\right)$.

and

$\nu \equiv\left(k / \beta_{0}\right), \quad \mu \equiv\left(\beta_{0} y_{0}\right), \quad p_{0} \equiv \tan \psi_{0}$,

$$
a_{0} \equiv\left(e E_{0} y_{0} / m c^{2}\right) \text {. }
$$

Before discussing the numerical solution of Eq. (28), it is instructive to consider an interesting special case in which the electron beam is longitudinally uniform, so that $\nu \equiv 0$, and the perturbation in the beam radius is small, i.e., $Y^{2} \ll 1$.

\section{Case I. Constant Velocity}

In the drift region, where there is no longitudinal electrostatic field $g_{2}=0$, under the conditions $\nu=0$ and $Y^{2} \ll 1, \mathrm{Eq}$. (28) reduces to

where

$$
\left(d^{2} Y / d x^{2}\right)+h_{1} Y+h_{0}=0
$$

and

$$
h_{1}=\left(p_{0}{ }^{2} / \mu^{2}\right)-\frac{1}{2}\left(\delta_{0}{ }^{2}-1\right)\left(1-\frac{3}{8} \mu^{2}\right)
$$

$$
h_{0}=\left(p_{0}^{2} / \mu^{2}\right)-\frac{1}{2}\left(\delta_{0}^{2}-1\right)\left(1-\frac{1}{8} \mu^{2}\right) \text {. }
$$

The complete solution of Eq. (29) consists of two parts-a complementary solution and a constant term representing the particular integral. The form of the complementary solution depends upon the algebraic sign of $h_{\text {. When }} h_{1}<0$, the complementary solution of Eq. (29) takes the form of a nonperiodic exponential function of $x$, which implies that the beam radius $y$ grows exponentially with the axial distance $\zeta$, so that the beam is continually spreading. However, when $h_{1}>0$, the complementary solution of Eq. (29) takes the form of a periodic (sinusoidal) function of $x$, so that the beam is rippling. For $h_{1}=0$, the solution of Eq. (29) has a quadratic dependence on $x$. Consequently the condition $h_{1}>0$ can be regarded as the condition for nonspreading of the drifting beam. The general solution of Eq. (29) for the case $h_{1}>0$ is given by

$$
\begin{aligned}
Y(x)=\left[Y_{0}^{\prime} /\left(h_{1}\right)^{1 / 2}\right] \sin \left(h_{1}\right)^{1 / 2} x \\
+\left[Y_{0}+\left(h_{0} / h_{1}\right)\right] \cos \left(h_{1}\right)^{1 / 2} x-\left(h_{0} / h_{1}\right)
\end{aligned}
$$

where $Y_{0}$ and $Y_{0}^{\prime}$ are, respectively, the normalized deviation in beam radius and the slope of the beam boundary at the entry to the drift region. When a parallel-flow beam is launched into the drift region, i.e., $Y_{0}=0$ and $Y_{0}^{\prime}=0$, Eq. (30) yields

$$
Y(x)=\left(h_{0} / h_{1}\right)\left[\cos \left(h_{1}\right)^{1 / 2} x-1\right] .
$$

In this case, the beam profile is characterized by two factors: (1) the amplitude of the beam ripple, and (2) the ripple wavelength (scalloping wavelength). Equation (31) indicates that $Y(x)$ varies between 0 and $-2\left(h_{0} / h_{1}\right)$. Since a laminar flow is being considered and $y \geqq 0,\left(2 h_{0} / h_{1}\right)$ must be less than unity. The conditions $h_{1}>0$ and $2 h_{0}<h_{1}$ can be combined to give

$$
\frac{1}{2} \mu^{2}\left(\delta_{0}^{2}-1\right)\left(1-\frac{3}{8} \mu^{2}\right)<p_{0}^{2}<\frac{1}{2} \mu^{2}\left(\delta_{0}^{2}-1\right)\left(1+\frac{1}{8} \mu^{2}\right) .
$$

Thus, when the beam parameters $\mu, \delta_{0}$, and $p_{0}$ are so chosen that condition (32) is satisfied, a rippling beam results. The profile given by Eq. (31) is illustrated in Figs. 1 for a conveniently chosen set of parameters. The normalized amplitude of the beam ripple denoted by $Y_{m}$ and the characteristic wavelength $\lambda$ are respectively given by

$$
\begin{aligned}
Y_{m}= & \left(h_{0} / h_{1}\right) \\
=\left[2 p_{0}^{2}-\mu^{2}\left(\delta_{0}^{2}-1\right)\left(1-\frac{1}{8} \mu^{2}\right)\right] /\left[2 p_{0}^{2}\right. & \left.-\mu^{2}\left(\delta_{0}^{2}-1\right)\left(1-\frac{3}{8} \mu^{2}\right)\right]
\end{aligned}
$$

and

$$
\begin{aligned}
\lambda & =2 / \beta_{0}\left(h_{1}\right)^{1 / 2} \\
& =2 \pi y_{0} /\left[p_{0}^{2}-\frac{1}{2} \mu^{2}\left(\delta_{0}^{2}-1\right)\left(1-\frac{3}{8} \mu^{2}\right)\right]^{1 / 2} .
\end{aligned}
$$

It should be observed that when $h_{0}=0, Y(x)=0$, so that the beam radius remains constant in the drift region (balanced flow). The condition for balanced flow, therefore, is written as

$$
p_{0} ?=\frac{1}{2} \mu^{2}\left(\delta_{0}^{2}-1\right)\left(1-\frac{1}{8} \mu^{2}\right) .
$$

Note that $\delta_{0}=\left(c / u_{z, 0}\right)$ and $\mu=\left(\beta_{0} y_{0}\right)$ can be expressed in terms of the input axial beam voltage $\Psi_{0,0}$ and the 


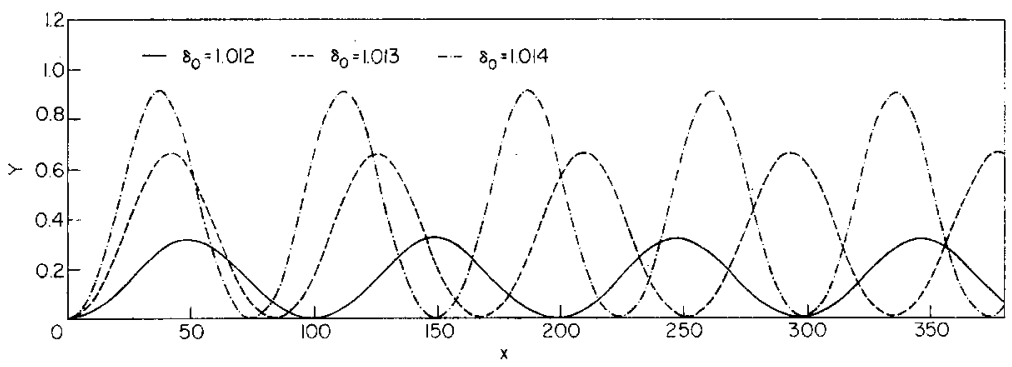

Fig. 2. General dependence of beam profiie on velocity. $\left(\mu=1.0, \quad p_{0}=0.1, \quad \nu=a_{0}=0\right)$.

axial beam current $\bar{I}_{0}$ as

and

$$
\delta_{0}=\left\{1-\left[1 /(1+Q)^{2}\right]\right\}^{-1 / 2}
$$

$Q \equiv\left(-e \Psi_{0,0} / m_{0} c^{2}\right)$

and

$$
D \equiv\left(\pi c^{3} m_{0} \epsilon / e\right)=-4.26 \times 10^{3} \mathrm{~A} .
$$

When $\bar{I}_{0}$ and $\delta_{0}$ are given in such a way that $\mu^{2} \ll 1$, then Eqs. (34) and (35) become, respectively,

and

$$
p_{0}^{2}=\frac{1}{2} \mu^{2}\left(\delta_{0}^{2}-1\right)
$$

$$
\mu^{2}=\left(\bar{I}_{0} / D\right)\left(\delta_{0}^{2}-1\right)^{1 / 2} \text {. }
$$

It is of interest to note that for most electron-beam devices the condition $\mu^{2} \ll 1$ is satisfied so that Eq. (36a) is applicable. In the case of Brillouin flow, Busch's theorem gives the angular velocity $\dot{\theta}$ at the entry to the drift region as the Larmor precession frequency, $\omega_{L}=\left(|e| B_{0} / 2 m_{0}\right)$, with $B_{0}$ denoting the uniform applied static axial magnetic flux density in the region under consideration. Consequently $u_{\theta}\left(y_{0}, 0\right)=y_{0} \omega_{L}$ and $\left(p_{0} / y_{0}\right)=G_{1}=\omega_{L} / u_{z, 0}$. Since $\delta_{0}=$ $\left(c / u_{z, 0}\right)$ and $\mu=\left(\beta_{0} y_{0}\right)$, Eq. (36a) can be written as

$$
\omega_{L}^{2}-\frac{1}{2} \omega_{p}^{2}\left[1-\left(u_{z, 0} / c\right)^{2}\right]=0 .
$$

It is observed that the last term of the left-hand side of Eq. (37) represents the relativistic focusing effect due to the $\theta$ component of magnetic field. When $\left(u_{z, 0} / c\right)^{2} \ll 1$, Eq. (37) reduces to $2 \omega_{L}^{2}=\omega_{p}^{2}$, which is the familiar equilibrium expression for nonrelativistic Brillouin flow.

\section{Case II. Accelerating Beam}

For the case in which a longitudinally uniform beam is accelerated by a uniform longitudinal static field, $\left(E_{0} \neq 0\right.$ and $\left.\nu=0\right)$, Eq. (28) reduces under the condition $Y^{2} \ll 1$ to

$$
\left(d^{2} Y / d x^{2}\right)+g_{2}(d Y / d x)+h_{2} Y+h_{2}=0,
$$

where

$$
h_{2}=\left(p_{0}^{2} / \mu^{2}\right)-\frac{1}{2}\left(\delta_{0}^{2}-1\right) .
$$

The complementary solution of Eq. (38a) has the form $e^{s x}$, where $s$ satisfies the following algebraic equation:

$$
s^{2}+g_{2} s+h_{2}=0 \text {. }
$$

In the accelerating region, $E_{0}$ must be negative and since $e$ is negative, $g_{2}>0$. Thus, Eq. (38b) has a pair of complex conjugate roots with a negative real part when $4 h_{2}>g_{2}{ }^{2}$. In this case, the complementary solution of Eq. (38a) is in the form of a damped oscillation such that the fluctuation in the beam radius is stable. On the other hand, when a longitudinal nonuniformity is permitted, $\nu$ must be different from zero. As an illustration, Eq. (28) is solved numerically for the input conditions $Y_{0}=Y_{0}^{\prime}=0$ and the results are shown in Figs. 2-4.

\section{DISCUSSION OF RESULTS}

The profiles of a uniform drifting electron beam, under the restriction $Y^{2} \ll 1$, are illustrated in Fig. 1 (a) for different values of axial beam velocity, $\delta_{0}=\left(c / u_{z}\right)$, and in Fig. 1(b) for various values of the input azimuthal to axial velocity ratio $p_{0}=\left(u_{0} / u_{z}\right)$. It is observed in Fig. 1 (a) that if $\Psi_{0,0}$ and $\bar{I}_{0}$ are adjusted so that $\mu$ is kept constant then the characteristic wavelength of the beam $\lambda$ increases as $\delta_{0}$ increases. This fact is also evident from Eq. (33). The normalized amplitude of the beam ripple $\left|Y_{m}\right|$ has its minimum value of zero when $h_{0}=0$, and for $h_{0}<0,\left|Y_{m}\right|$ increases as $\delta_{0}$ increases. The plots of Fig. 1 (b) indicate that for given values of $\Psi_{0,0}$ and $\bar{I}_{0}, \lambda$ decreases as $p_{0}$ increases, and thus the pinch effect increases as $p_{0}$ increases. The variation of $Y_{m}$ and $\lambda$ with the system parameters for a drifting beam can be easily studied by inspecting Eqs. (33).

The profile of a uniform beam in a drift region without the restriction of $Y^{2} \ll 1$ is shown for various

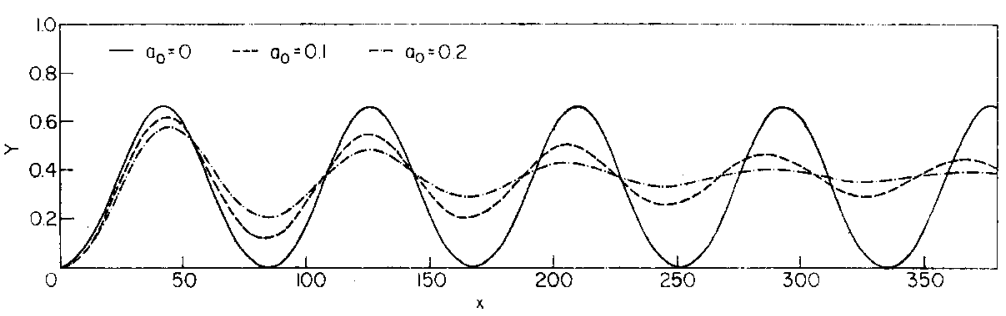

FIG. 3. Effect of a longitudinal accelerating static electric field. $\left(\delta_{0}=1.013, \mu=1.0\right.$, $p_{0}=0.1, \nu=0$ ). 
Fig. 4. Effect of a longitudinal inhomogeneity. $\left(\delta_{0}=1.013, \mu=1.0, p_{0}=0.1, a_{0}=\right.$ $0.1)$.

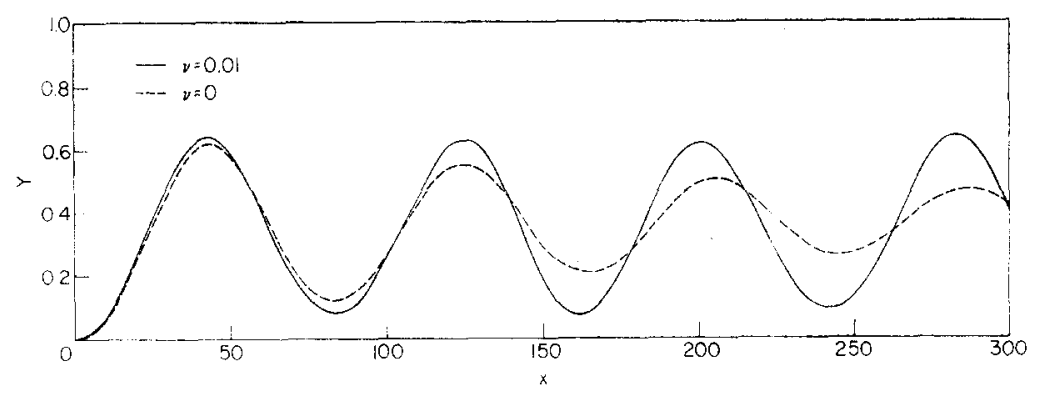

values of velocity $\delta_{0}$ in Fig. 2 . It is observed that the maximum beam radius is an almost periodic function of the axial distance and the amplitude of oscillation in the beam boundary decreases as $\delta_{0}$ decreases, i.e., as the axial beam velocity approaches the velocity of light. The remarks made above in regard to Fig. 1(a) are also applicable to Fig. 2. The effect of a longitudinally uniform static accelerating electric field $E_{0}$ on the profile of a uniform beam is shown in Fig. 3. It is noteworthy that the fluctuation in beam radius is damped as $E_{0}$ is introduced since $a_{0}=\left(e E_{0} y_{0} / m c^{2}\right) \neq 0$. The maximum normalized deviation in beam radius decreases as $E_{0}$ increases and the amplitude of oscillation in beam radius decreases as $x$ increases. This is consistent with the observation made in Fig. 2 since, as the beam is accelerated and at large value of $x$, the axial beam velocity is increased so that $\delta_{0}$ is decreased, thus reducing the amplitude of oscillation of the beam boundary. Finally, the profile of a beam with a small nonuniformity in the accelerating region is illustrated in Fig. 4. The nonuniformity has the effect of increasing the amplitude of oscillation of the beam boundary.

\section{CONCLUSIONS}

In the present paper the analysis of relativistic electron flows has been generalized to account for radial variations in the electron velocity. The condition for nonspreading of a laminar-flow drifting electron beam is given by inequality (32), which is expressed in terms of the beam parameters $p_{0}, \mu$, and $\delta_{0}$, which are related to the ratio of transverse to longitudinal velocity at the input, the beam current, and the axial beam voltage. It is shown that for the case $\mu^{2} \ll 1$, which is rather common in many experimental systems, the equilibrium condition for Brillouin flow with the relativistic correction is given by Eq. (37). The derived condition (37) implies that for a fixed $\omega_{p}$ the higher the axial velocity of the beam the less applied axial magnetic field that is needed to obtain a perfectly balanced flow. In the present investigation, since it is assumed that the transverse velocity is much smaller than the axial velocity, the axial component of self-induced magnetic field would be much smaller than the $\theta$ component of self-induced magnetic field and consequently the dominant self-focusing effect is due to the Lorentz force from the $\theta$ component of selfmagnetic field and the axial beam velocity.
If a linear beam is launched at the entry of the drift region, in which case $p_{0}$ is zero, then from Eq. (34) it is necessary that either $\delta_{0}=1$ or $\mu^{2}=8$ for the beam to be perfectly balanced. However, for normal laboratory operating conditions, the latter condition, i.e., $\mu^{2}=8$, is rarely satisfied. Consequently, a perfectly balanced flow would not likely be obtained unless the axial beam velocity is nearly equal to the speed of light in vacuum.

Nonzero values of $\nu=\left(k / \beta_{0}\right)$ indicate the existence of a nonuniformity along the beam. The cause of the nonuniformity may be due to various factors, e.g., for short beams the beam termination will effect the overall beam configuration due to reflections. Also when a static spatially periodic electric field is used in the focusing of the beam, $k$ can be determined from the spatial periodicity of the applied static electric field.

The system parameters used for Figs. 1-4 are conveniently chosen to illustrate the method of analysis and generally correspond to the physical conditions existing in accelerator devices rather than those of microwave beam devices. However, it is not difficult to make similar calculations for the parameters which represent closely the physical condition encountered in any experimental system.

The method of analysis developed here can be extended to include the effects of positive ions which may be present when a partially neutralized beam is considered, provided that the potentials $\Psi$ and $A$ in the system are properly modified.

\section{LIST OF SYMBOLS}

$T=$ the particle kinetic energy,

$\epsilon_{0}=$ the particle rest energy,

$M_{0}=$ the integration constant [see text following Eq. (9) ],

$M_{1}=$ the integration constant [see text following Eq. (23)],

$M_{2}=$ the integration constant [see text following Eq. (24)]

$M_{3}=$ the integration constant [see text following Eq. (23)],

$K_{0}=$ the equivalent angular momentum of a particle,

$\mathbf{A}=$ the vector potential,

$\omega_{L}=$ the Larmor angular precession frequency,

$\omega_{p}=$ the electron radian plasma frequency,

$\mathbf{p}=$ the particle linear momentum,

$\psi=$ the scalar potential. 